\title{
Accuracy and precision of the CSLT measurement system: An experiment to defect diagnoses in bored piles
}

\author{
Zezhong Wang ${ }^{1, *}$, Eric Tak Cho Ho ${ }^{1,2}$ and Inez Maria Zwetsloot ${ }^{1}$ \\ ${ }^{1}$ Department of Advanced Design and Systems Engineering, City University of Hong Kong, Hong Kong, People's Republic of \\ China \\ ${ }^{2}$ FT Laboratories Ltd., Fanling, Hong Kong, People’s Republic of China
}

\begin{abstract}
A new measurement system called Crosshole Sonic Logging Tomography (CSLT) provides information on the size, shape, and orientation of defects in a bored pile. The CSLT measurement system has not (yet) been accredited in Hong Kong for foundation testing. Bored piles in Hong Kong are generally wide and deep. Existing measurement accuracy studies do not consider this large type of bored piles. The objective of this research is to quantify the measurement accuracy and precision of the CSLT method for large diameter bored piles (the most common pile type for public housing projects in Hong Kong). A test pile was constructed with known defects and perform experiments with a CSLT measurement system to quantify its accuracy and precision. CSLT is found to be accurate in detecting shape, size, and location of large defects but small defects close to the tube are difficult to detect. Generally speaking, CSLT has satisfactory accuracy and precision for practical use. The use of CSLT can be considered as a feasible method in defect diagnosis of bore piles in Hong Kong.
\end{abstract}

KEYWORDS Concrete testing; ultrasonic; crosshole tomography; foundation testing; measurement accuracy and precision; CSLT; CSL; bored pile

CONTACT Zezhong Wang zezhowang3-c@my.cityu.edu.hk

Received 8 March 2021

\section{Introduction}

In Hong Kong the reliability of construction testing results has become a subject of ongoing intense discussion due to a mega scandal revealed recently at the Hong Kong-Zhuhai-Macau bridge project. The Independent Commission Against Corruption arrested 21 staff members of a construction contractor, who were suspected of corruption and having submitted fraudulent concrete compression test reports (Leung C, 2017). It is a general expectation from the Hong Kong public that the construction industry in Hong Kong is starving for advancement in technology and committed to quality improvement. One opportunity for such innovation will be studied in this article. The accuracy and precision of a new measurement system for foundation testing, the Cross-hole Sonic Logging Tomography (CSLT) test will be evaluated.

Foundation testing, including bored pile testing, is an important aspect of structural engineering. In Hong Kong, bored piles are typically large and deep. Defect can arise during the concrete pouring of bored piles. Detecting such defects is important as it allows the contractor to investigate and resolve the defect thereby increasing the reliability and safety of the overall structure. In Hong Kong, bored piles need to be tested by certified testing laboratories according to the adopted standard (ASTM D6760). The standard prescribes the use of the Crosshole Sonic Logging (CSL) method. This method is an often-used method worldwide for assessing concrete quality in a bored pile (Hajduk et al,. 2004).
The standard one-dimensional CSL test (ASTM 676016 2016) is a non-destructive method of testing concrete homogeneity and locating irregularities in bored piles. Results of CSL testing are typically displayed with onedimensional (1D) representation. When a defect or anomaly is detected, the only information about defect location is the elevation within the shaft where this feature exists. Therefore, the interpretation of test measurements by the CSL method requires some judgment and experience. This traditional non-destructive method of assessing the integrity of deep foundation elements does not provide true threedimensional (3D) representation of the subsurface site conditions. Therefore, the CSL test results do not allow for the quantification of the severity of a defect or defects located within the same cross-section.

An emerging innovative technology is the socalled Crosshole Sonic Logging Tomography (CSLT) which provides a visualisation of the shape, size, and location of irregularities in the shaft (Jalinoos et al., 2005). Tomography or tomographic analysis is a mathematical procedure that is applied to CSL data to create a two- or three-dimensional visual image of the bored pile. Thereby, the CSLT provides much more qualitative and quantitative information about the bored piles' integrity. Specifically, it allows for the lateral and vertical extent of possible defects/ flaws to be examined, thereby giving the engineer more information to decide on an appropriate follow-up action. The CSLT measurement system has not been accredited by Hong Kong Accreditation Service (The Hong Kong Laboratory Accreditation Scheme, HOKLAS ${ }^{1}$ ) for using in foundation testing in Hong Kong. 
The aim of this study is to quantify the accuracy and precision of the CSLT measurement system. A 1.2-metre diameter test pile shaft with three known defects has been constructed and experiments with CSL and CSLT measurement systems were conducted. Experimental data was used to quantify the accuracy and precision of the CSLT test. The data, which are in the form of 2D images, are processed by feature extraction. The images were summarised by using four performance criteria and compute accuracy and precision based on seven repeated measurements. An additional experiment was performed to determine how many days after concreting the CSLT measurements gives accurate results.

It is the aim to apply for HOKLAS accreditation of the CSLT test. The presented measurement study is an important aspect of the accreditation process. The possible adoption of the CSLT test can bring innovations to the construction industry by promoting it as a standard method in the construction industry of Hong Kong for evaluating defects in deep foundations.

\section{Literature review}

During the construction of deep bored piles imperfections may occur, to ensure the shaft integrity it is common to perform post-construction measurements. Likins et al. (2012) briefly reviewed available techniques for evaluating deep foundations quality, including the CSL tests that are commonly used to test bored piles. To quantify and evaluate the integrity of bored piles, Likins et al. (2007) proposed numerical thresholds for the two major results from CSL tests, first arrival time (FAT) and energy level. White et al. (2008) illustrated that the CSL provides ability to quantify a defect in the shaft and allows for determination of shaft quality. Various studies have shown that the CSL method can detect and locate defects in bored piles accurately, see Williams and Jones (2008), Amir and Amir (2009), Islam et al. (2015), Sibit and Handayani (2016), and Lozovsky et al. (2020) for reference.

One limitation of the widely used one-dimensional CSL is that it can only locate defects by depth, but the size and shape of defect are unclear. Deep Foundation Institute (2019) published a white paper to review the state and experience of applying CSL test in the past 20 years. They proposed a set of improved CSL rating criteria and recommended for additional assessment. Finno and Champy (1997) suggested the smallest defect size that can be reasonably detected by CSL is about one wavelength of the ultrasonic signal and the frequency of the ultrasonic signal can vary from $35 \mathrm{kHz}$ to $60 \mathrm{kHz}$, a higher detection accuracy will be obtained with a higher frequency. Although high frequencies permit more accurate detection of a defect, but the signal absorption will also be high (Mor, 1991). In this study, Cross Hole Ultrasonic Monitor (CHUM) which has a frequency of $50 \mathrm{kHz}$ was adopted. Likins et al. (2007) and Amir and Amir (2009) suggested to use three-dimensional tomography analysis to enhance the flaw detectability after identifying an anomaly. Once the anomaly zone is detected, the horizontal cross-sectional images at the same depth can further visualise and quantify the abnormal zone. Chernauskas and Hajduk (2009), and Zhussupbekov et al. (2019) used cross-sectional images to refine the CSL tests result.

Instead of using the tomography as a complement of CSL tests, Jalinoos et al. (2005) proposed a clear methodology from detecting the anomalies to assessing the integrity of the bored pile based on both CSL and CSLT data. The CSL tests were used to correctly identify and independently verify anomalies in bored pile, and CSLT technique was used for imaging of the shaft's interior. To examine how well the CSLT results represent the velocity field (especially the defect velocity), they compared the percentage drops in minimum defect velocity to the shaft velocity. They concluded that the tomography slightly overestimates the size and elongates the defects. For more details about this work, refer to Jalinoos et al. (2005).

Many authors have studied the accuracy of CSLT methodology, Hajali and Abishdid (2014) proposed a signal processing method called frequency tomography analysis (FTA) to detect and locate the abnormality in bored piles. FTA can increase the location accuracy and characterise the feature of abnormality for further analysis. Amir and Amir (2009) used the increase of FAT and attenuation to quantify the result of tomography methods with different dimensions. Attenuation is recommended for 3D tomography because it can reveal the flaws more accurately. Lozovsky et al. (2020) inversed the cross-hole ultrasonic tomography data to reconstruct the position of defects and compare it with the actual position to evaluate the result of CSLT methods. Jalinoos et al. (2005) performed a single CSLT on a pile with a known defect and report the percentage drop in the minimum wave speed, which only reflects the size of defect and ignores the location. Hence, the accuracy and precision of the CSLT method on bored piles is unknown. To fill this gap and promote the application of CSLT technique in Hong Kong, an experiment is proposed in this paper.

\footnotetext{
${ }^{1}$ HOKLAS is an accreditation scheme operated by the Hong Kong Accreditation Service (HKAS). The scheme is open to any Hong Kong laboratory that performs objective testing and production of reference material falling within the scope of the Scheme and meets the HOKLAS criteria of competence. HKAS accreditation is granted for specific activities. An accredited organisation can issue HOKLAS endorsed reports / certificates only for tests and calibrations and reference materials which for it is accredited.
} 


\section{Experimental set up}

This section describes the CSLT method and the experimental setup, including the test pile that was constructed, the data collection plan, sample data, and the features used to quantify accuracy and precision. This section starts with a short description of the CSL method which is used to determine the depth of a potential defect and is the initial step performed before the CSLT is run.

\subsection{CSL method description}

The CSL test is a non-destructive method of testing concrete homogeneity and locating irregularities in bored piles. It is a mandatory acceptance test for all projects involving bored piles construction with the Hong Kong Housing Authority (HKHA). The adopted standard for the test is ASTM D6760 and the test shall be carried out only by approved personnel of a HOKLAS accredited laboratory. The CSL test is performed by sending and receiving ultrasonic pulses through concrete between emitter and receiver probes placed at the same level in parallel water-filled tubes. To begin the test, the probes are positioned at the bottom of a pair of access tubes in the pile, and then raised simultaneously. The CSL equipment records the signal response and measurement depth for the entire logged length. The primary tool for presenting and assessing the results of the CSL tests is the CSL log, a graphical presentation of the signal arrival times and energy levels for a single tube pair for the entire scanned length of pile. The CSL log/profile shows the following transmission characteristics of the concrete between a pair of tubes as a function of depth:

- Travel time of the ultrasonic pulse from the source to receiver probe.

- Amplitude (or energy) of received signal.

The evaluation of pile integrity is based on:

- Consistency of the signal arrival times from the top to bottom of the pile.

- Signal energy.

For homogeneous concrete, the travel path of the signal is a straight line between the probes. Thus, a CSL $\log$ for piles with homogeneous concrete shows consistent transmission time and received signal amplitude (energy) with depth. On the other hand, if a material with a much lower transmission velocity than concrete is placed between the probes, the signal will diffract around it such that the travel path is no longer straight, resulting in the increase in transmission time and a weakening of the signal energy. Thus, a delay in signal travel time and the weakening of signals observed in the CSL logs can be indicative of irregularities in the concrete between the tubes.

\subsection{CSLT method description}

Tomography or tomographic analysis is applied to CSL data that combines FAT data from the scans of all pairs of CSL tubes to provide a 2D or 3D visual image of the pile shaft. Specifically, it allows for the lateral and vertical extent of possible defects/flaws to be examined.

The CSLT method uses the same equipment and access tubes as the CSL method. For CSLT testing, wave speed data are collected for many receivers and source combinations at different depths, in contrast to CSL testing which used probes at the same depth only. CSLT employs inversion procedure on the FAT data of compressional wave energy. Firstly, the test zone is divided into many cells with assumed slowness values (inverse of velocity). Then the expected travel times along the test paths are calculated and compared with the measured travel times. Mathematical models are used to redistribute errors along the individual cells. Finally, the process iterates until the measured travel times match the assumed travel times within an assumed tolerance. The outcome is a $2 \mathrm{D}$ or $3 \mathrm{D}$ image of the internal structure of foundation which reveals good and defective zones.

(a)$$
\# 2
$$

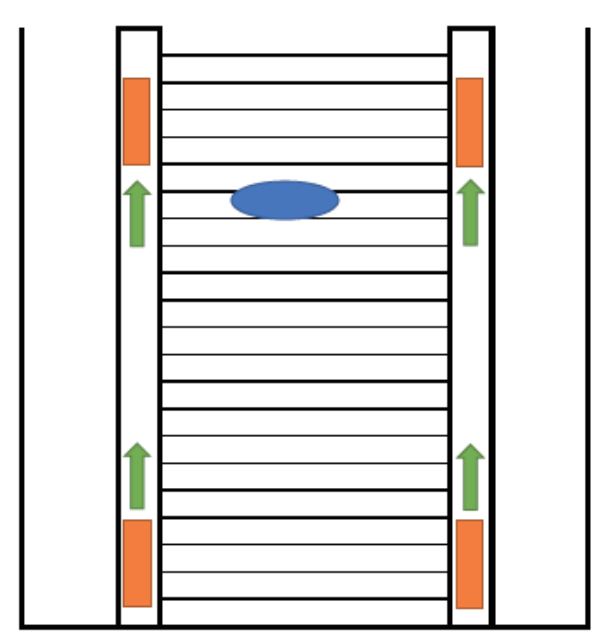

(b)

$$
\# 2
$$

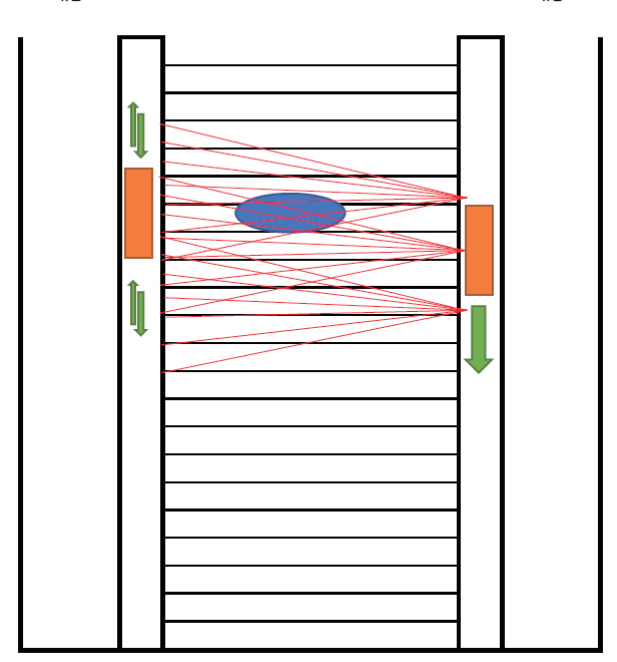

Figure 1. (a) CSL procedure with both probes pulled horizontally; and (b) CSLT procedure with probes being offset in many different angles. 
In Hong Kong, the CSLT method is not currently adopted as a mandatory test procedure for bored piles which have been identified with defects by the CSL method. The CSLT procedure is described in the ASTM D6760 CL.7.8.1 and visualised in Figure 1(b). The CSLT is described as additional step that can be carried out during the CSL test, when the ultrasonic profile indicates an anomaly. The suspect anomaly zone may be further investigated by special test procedures such as the tomographic techniques. The clause also mentioned specifically that the probes shall be lowered to a depth of at least $1 \mathrm{~m}$ below the anomaly and raised to a depth of at least $1 \mathrm{~m}$ above the anomaly. The continual clause of CL.7.8.2 instructs the operator to repeat the steps for the remaining pairs of access tubes after the additional steps of CSLT has been completed.

The CSL and CSLT tests for this paper were performed using the Cross Hole Ultrasonic Monitor (CHUM) manufactured by Piletest Inc..

\subsection{Test pile}

In order to evaluate the accuracy and precision of the CSLT method, a test pile shaft with known defects was constructed to simulate a bored pile foundation. The diameter of the test pile shaft was $1.2 \mathrm{~m}$, and it was $4.2 \mathrm{~m}$ in height. The pile was constructed with $45 \mathrm{MPa}$ reinforced concrete. Six steel CSL access tubes with a diameter of 40 $\mathrm{mm}$ were installed at equal spacing around the inner rim of the pile shaft casing, each CSL access tubes was $100 \mathrm{~mm}$ away from the inner surface of the pile shaft. The CSL access tubes are taller than the pile shaft for $0.2 \mathrm{~m}$. Six tubes were installed as it is the most abundant scenario for large diameter drill shaft being used for HKHA's construction projects.

There were three built-in defects preinstalled in the test pile shaft. The type and sizes of the defects were carefully selected with the consideration that a defect, located halfway between two access tubes, is only detectable if its size exceeds about $1 / 3$ of tube spacing or about $10 \%$ of the pile's cross-section (Amir and Amir, 2009). The first defect (defect 1) as shown in Figure 2(b) is a cubic steel hollow box with a side length of $0.3 \mathrm{~m}$. It is placed at the centre of the test pile shaft at a depth of $1 \mathrm{~m}$ between tubes 1 and 4 . The second defect (defect 2) as shown in Figure 2(c) is a $0.2 \mathrm{~m}$ long bandage wrapped around the CSL access tube 3 at a depth of approximate $2 \mathrm{~m}$. The bandage is used to simulate an imperfection of bonding between the concrete and the CSL access tube. The third defect (defect 3 ) as shown in Figure 2(d) is a cubic hollow steel box with side length $0.2 \mathrm{~m}$ and is placed near to the 6 th access tube at a depth of around $3 \mathrm{~m}$. Figures 2 shows the design of the test pile and the pile while concreting, Figure 2(b) clearly shows defect 1 and some reinforcements in the shaft. Note that the size of these defects would be classified as category A and $\mathrm{B}$ by the technical specification of non-destructive pile testing contract of HKHA (Cl. D10). These defects would be considered minor structural defects. (a)
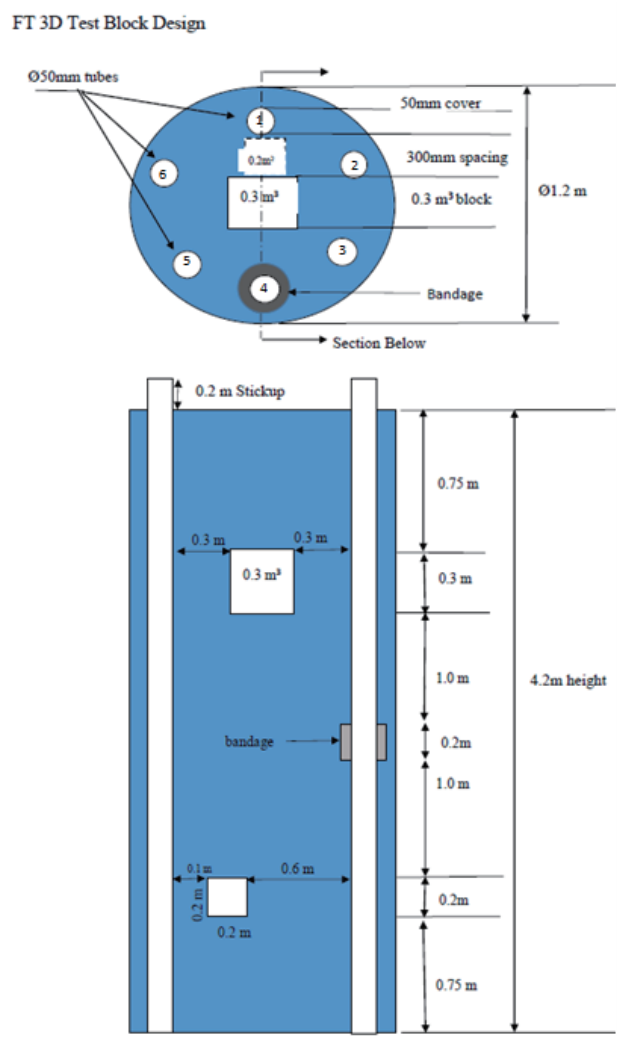

(b)

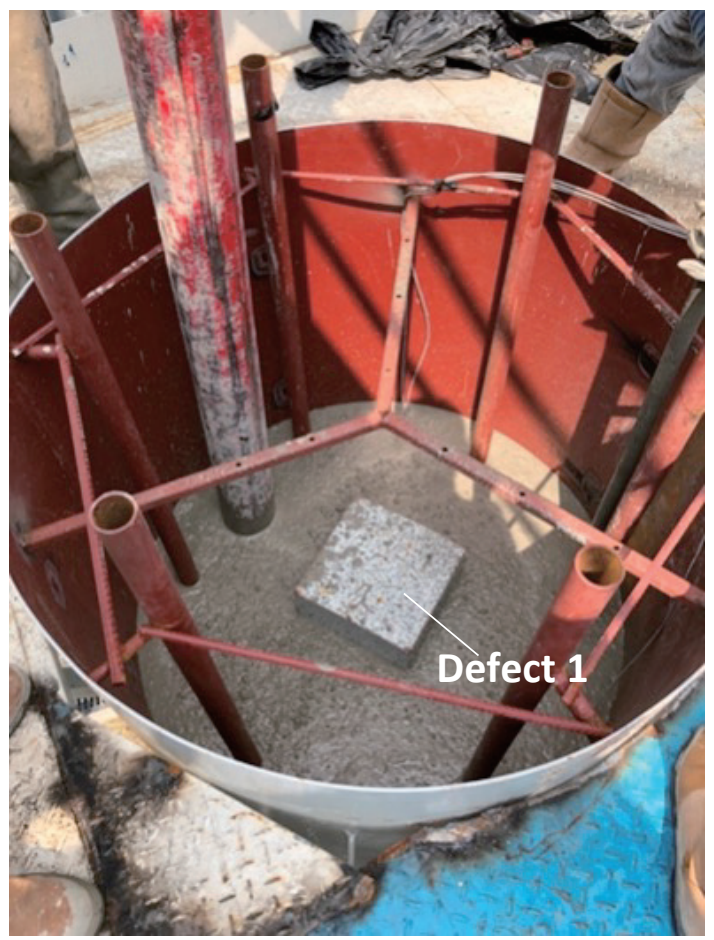


(c)

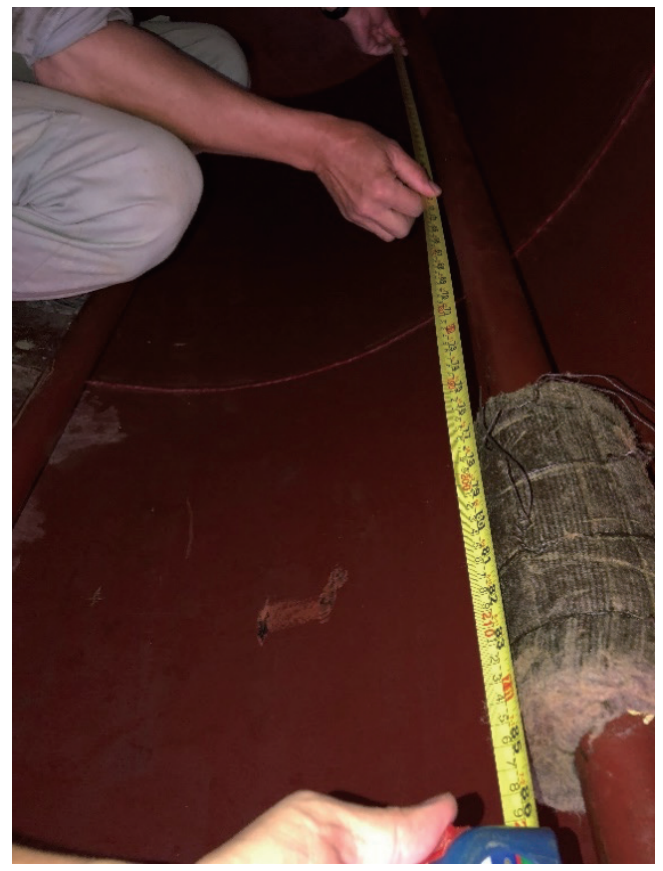

(d)

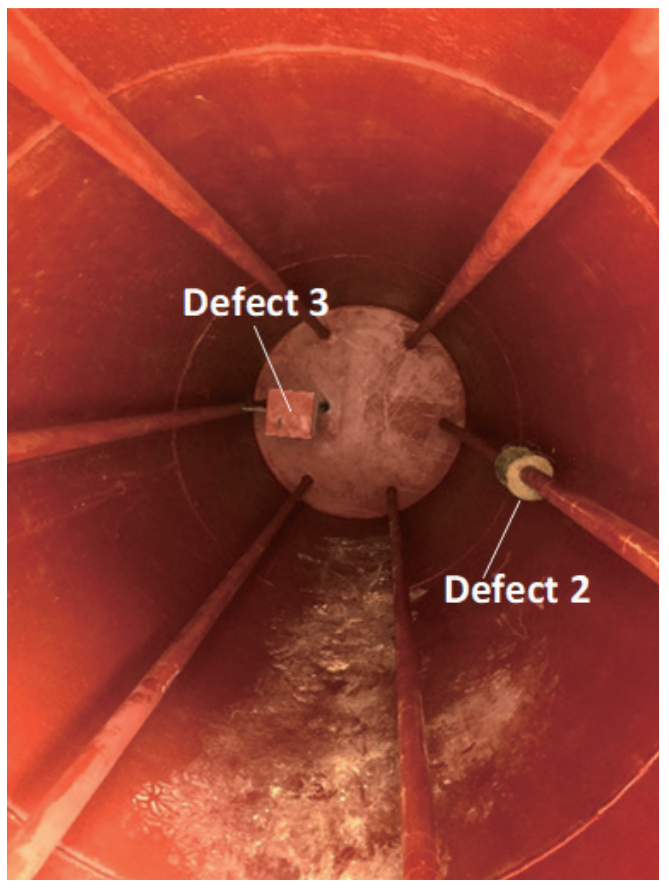

Figure 2. (a) Test pile shaft design; (b) pouring the concrete pile; (c) close-up of defect 2; and (d) defect 2 and defect 3.

\subsection{Data collection plan}

To evaluate the accuracy and precision of the CSLT method, the following experiment was set up.

The measurement procedure consists of two steps, (1) performing the CSL test; (2) performing the CSLT test at the depth where a possible defect is indicated by CSL. Right before the test is conducted, the CSL access tubes were checked for free access and filled with water to obtain good acoustic coupling. 15 CSL data profiles (e.g., tubes
$1-2,2-3$, and so on) were collected between the access tubes inside the concrete.

The FAT and energy data can be viewed on the computer screen at real-time during the data collection. According to the principle proposed by HKHA, when the delay of FAT is greater than $20 \%$, further investigation is recommended, of which core drilling is generally used but the CSLT method would be a feasible alternative. The FAT is detected by a built-in algorithm named "Automatic First Arrival Time Picking" in the CHUM software. Amplitudes vector V(i) is the input while FAT is the output. When a defect in the tested medium blocks the signal, it absorbs some (or all) of the transmitted energy, and a lower energy value is detected by the receiver. Some defects such as necking can significantly reduce the received energy but do not change the FAT since a direct path between the transmitter and the receiver still exists.

Energy is calculated as the sum of the absolute voltage values along the received pulse:

$$
\mathrm{E}=\sum_{i}|V i|
$$

Relative Energy (RE) is the relative signal strength of the pulse arriving at the receiver compared with a reference signal strength. The test bored pile was originally constructed in July 2018, CSL and CSLT were performed on eight selected days after the concrete was placed in the test pile: the $2^{\text {nd }}, 4^{\text {th }}, 7^{\text {th }}, 10^{\text {th }}, 14^{\text {th }}, 18^{\text {th }}, 23^{\text {rd }}$ and $28^{\text {th }}$ day after concreting. In February 2021, seven repeated CSL and CSLT data were measured by a team of two people including a field technician and a qualified CSL test operator accredited by HOKLAS. These data were collected within three consecutive days using the same equipment with the same procedure. In Section 4, both the repeated measurement and the measurements after concreting to study the accuracy and precision of the CSLT are analysed.

Once an abnormality is detected by CSL, the CSLT method is applied to the corresponding depth. The CSLT detection ranges shall cover $1 \mathrm{~m}$ below the anomaly and $1 \mathrm{~m}$ above the anomaly (e.g. CSL identified am anomaly at a depth of $3.2 \mathrm{~m}$ from pile top, the depth range for CSLT scanning shall be from $4.2 \mathrm{~m}$ to $2.2 \mathrm{~m}$ from pile top). The depth steps are $10 \mathrm{~cm}$ for all combinations of access tube (i.e. 15 profiles).

\subsection{Sample data}

For each CSL profile, a graphical presentation of the signal arrival times and energy levels for the entire scanned length of pile was obtained, two examples at $28^{\text {th }}$ day are displayed in Figure 3. Figure 3(a) shows the profile of tubes 3-6, and Figure 3(b) is from tubes 5-6. The latter shows a clear profile with no visible anomaly, whereas the profile of 3-6 shows anomaly at a depth of approximate $1.0 \mathrm{~m}$, $2.2 \mathrm{~m}$ and $3.3 \mathrm{~m}$, where the lines cross. These anomalies are recorded as classes $\mathrm{B}$ and $\mathrm{C}$ which corresponds to yellow zone and red zone on the left side of the profile. Note 
that the profile also shows possible defects at the top and bottom of the pile, they can both be ignored as the defects at the bottom are imperfections for the construction of the test pile and the defects at top of the piles are caused by the protruding access tubes at the top of the test pile where there is no concrete. (a)

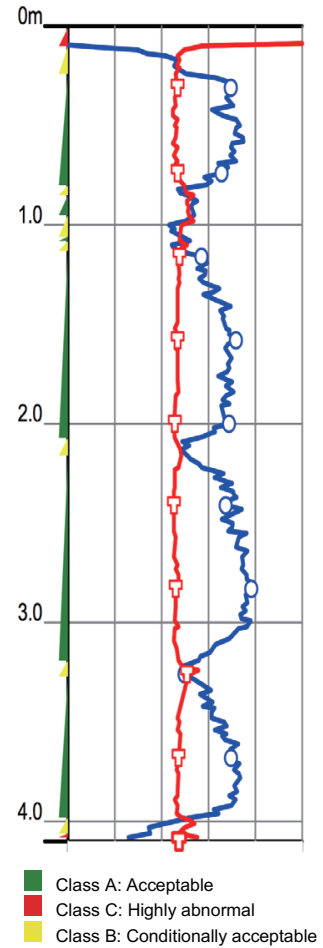

(b)

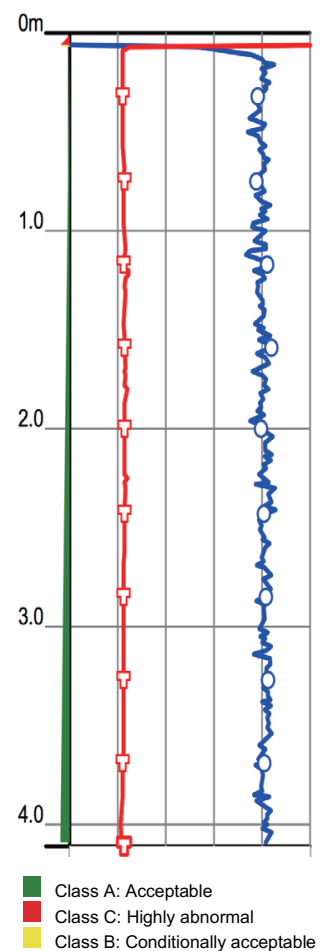

Figure 3. (a) CSL profile of tubes 3-6 with anomalies detected; and (b) CSL profile of tubes 5-6 with no significant anomaly detected.

Figure 4 shows a $28^{\text {th }}$ day aged $3 \mathrm{D}$ tomography view of the test pile created by the CSLT software. A continuous spectrum from red to blue is used to represent estimated wave speeds which are related to estimated concrete quality. The relationship is shown in the left palette in Figure 4. The lower wave speed, which is shown in red, indicates bad quality. Blue, green and grey represents good concrete quality with high wave speed.

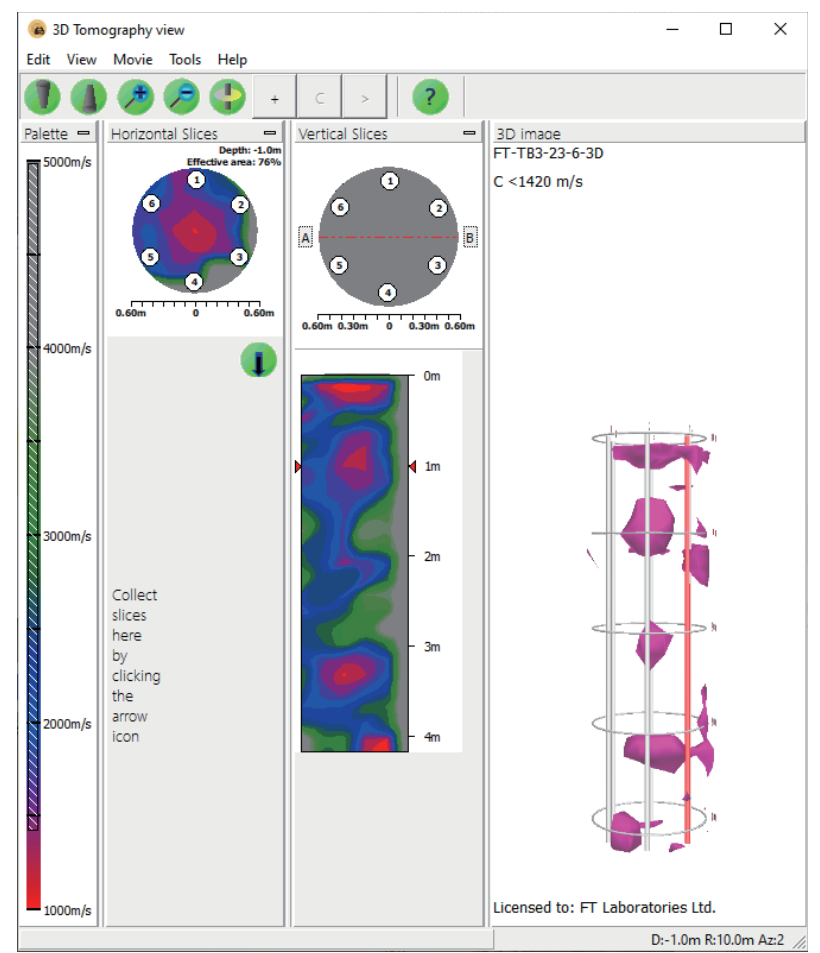

Figure 4. 3D tomography of the test pile created by the CSLT method.

A built-in horizontal slicing tool of the 3D tomography programme was used to capture cross-section images at the depths with suspicious defects. These depths coincide with the CSL profiles and the predefined defects.

These slices were exported for further analysis to be carried out in the following sections. Figure 5 shows three examples of horizontal slices extracted from different depths.
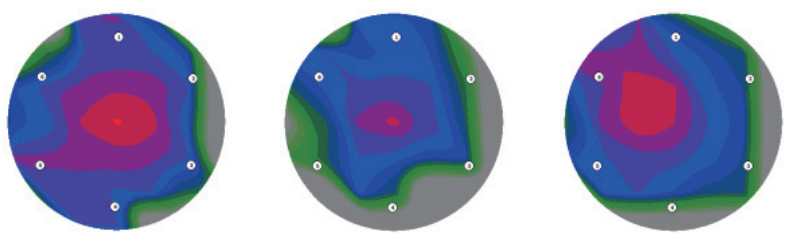

Figure 5. Cross-section CSLT images of test pile at $1.0 \mathrm{~m}$, $2.2 \mathrm{~m}$, and $3.3 \mathrm{~m}$ depth from top.

\subsection{Four performance criteria}

To obtain an overall evaluation of the CSLT method, It was proposed to extract three novel features from the crosssections, and combine them with one inherent measurement from the tomography software to quantify the character of the cross-section images. By comparing the extracted features with the real defects, the accuracy and precision of CSLT method can be evaluated comprehensively.

The default measurement from the tomography software is the effective area, which represents the 
percentage of cross-sectional area with estimated wave speed greater than an effective wave speed (EWS), derived from an average wave speed (AWS) calculated by the software. A lower wave speed may indicate poorer quality concrete, therefore larger effective area reflects good quality. This measurement reflects the relative size of potential defect in the cross section. An obvious limitation of effective area is that the specific location and shape of the defective area is not known.

In the result of the CSLT method, different colours correspond to different wave speed, and lower wave speed indicates potential defects. To quantify the size and shape of potential defects on cross-sectional images, a threshold of $1,250 \mathrm{~m} / \mathrm{s}$ was determined to extract the defective area according to the standard used in the software. All pixels that present the colour with wave speed lower than $1,250 \mathrm{~m} /$ $\mathrm{s}$ are selected. Since the position of each pixel is recorded, this group of pixels can represent not only the size but also the shape and the location of the detected defect. $R$ is used to denote the selected area. The size, shape, and location of the real defects on the cross-sectional images can be represented by a group of pixels $(D)$.

Based on the groups $R$ and $D$, two metrics, the size ratio and the overlapping rate are defined. The size ratio represents the relative size difference between detected defects and real defects. It is calculated by size ratio $=N(R) / N(D)$, where $N(R)$ and $N(D)$ are the number of pixels in $R$ and $D$. A size ratio greater than one indicate that the CSLT method overestimates the defect size, otherwise it underestimates, a size ratio of 1 is perfect detection.

By considering the position of pixels in $R$ and $D$, the overlapping rate can be calculated by overlapping rate $=N(R \cap D) / N(D)$, where $N(R \cap D)$ is the number of pixels that are contained in both groups. This measurement reflects the proportion of detected defective area that can be explained by the real defects. An overlapping rate close to one indicates that the CSLT method can detect the size of the defect correctly.
Finally, the location deviation to measure the shift between detected and real defects was considered. The lowest wave speed on each cross-sectional image is used to define and calculate the centre of defects, the selected pixel group is defined as $L$. The centre of detected defects and real defects, $\left(x_{L}, y_{L}\right),\left(x_{D}, y_{D}\right)$ are calculated by taking average position of pixels in group $L$ and $D$ respectively. Then the location deviation which is the distance between the detected and real defect centre can be computed by $\sqrt{\left(x_{L}-x_{D}\right)^{2}+\left(y_{L}-y_{D}\right)^{2}}$. A value of location deviation equal to zero indicates that the centre of the detected defects coincides with the centre of actual defects.

\section{Results and analysis}

\subsection{CSL results}

The results of the CSL measurements on the test pile are summarised in Table 1 . There were no anomalies detected for most profiles (1-2, 2-3, 3-4, 4-5, 5-6, 1-6, 2-6, $2-4,4-6,3-5)$. The CSL profile associated with tubes 1-4, $2-5,3-6,1-3$ and 1-5 shows possible defects around the depth of the real defects. It is not surprising that only the cross-sectional CSL profiles show defects, as the placed defects at $1 \mathrm{~m}$ is located in the centre part of the pile and defects at $3.3 \mathrm{~m}$ is a bit off-centre and near to tube 6 . However, the defect at $2.2 \mathrm{~m}$ is located at the side of the pile around tube 3 . It is therefore somewhat surprising that profiles 1-4, 2-5, and 1-5 indicate defects at around $2.2 \mathrm{~m}$. One possible explanation for this is the reinforcement frame that was installed near the $2.2 \mathrm{~m}$ defect. Overall, the depth of the detected anomaly among all profiles corresponds to the depths of the installed defects.

\subsection{CSLT results}

Following to the CSL results as summarised in Table 1, the results indicated possible defects at the depths of $1.0 \mathrm{~m}$,

Table 1. CSL results for bore pile with 3 defects at the depths of $1.0 \mathrm{~m}, 2.2 \mathrm{~m}$, and $3.3 \mathrm{~m}$.

\begin{tabular}{|l|l|l|}
\hline \multicolumn{2}{|l|}{ Profiles } & $\begin{array}{l}\text { Depth defect } \\
\text { detected (m) }\end{array}$ \\
\hline $1-3$ & 2.2 \\
\hline $1-4$ & & $0.8-1.1 ; 2.1$ \\
\hline $1-5$ & & 2.1 \\
\hline $2-5$ & & $0.8-1.1 ; 2.1 ;$ \\
& 3.2 \\
\hline $3-6$ & $0.9-1.1 ; 2.1 ;$ \\
\end{tabular}

\begin{tabular}{|l|l|l|}
\hline \multicolumn{2}{|l|}{ Profiles } & $\begin{array}{l}\text { Depth defect } \\
\text { detected }(\mathrm{m})\end{array}$ \\
\hline $1-2$ & & none \\
\hline $1-6$ & & none \\
\hline $2-3$ & & none \\
\hline $2-4$ & & \\
\hline $2-6$ & & none \\
\hline
\end{tabular}

\begin{tabular}{|l|l|l|}
\hline \multicolumn{2}{|l|}{ Profiles } & $\begin{array}{l}\text { Depth defect } \\
\text { detected }(\mathrm{m})\end{array}$ \\
\hline $3-4$ & & none \\
\hline $3-5$ & & none \\
\hline $4-5$ & & none \\
\hline $4-6$ & & none \\
\hline
\end{tabular}


$2.2 \mathrm{~m}$ and $3.3 \mathrm{~m} .15$ separate CSLT tests were performed on a depth of $1.0 \mathrm{~m}, 2.2 \mathrm{~m}$ and $3.3 \mathrm{~m}$. which include seven repeated measurements when the concrete was properly cured and 8 after concreting measurements when concrete was still curing with preset defects.

\subsubsection{Seven repeated experiments}

For each of the seven repeated experiments, three cross-section images were obtained from the software on the depths indicated by the CSL and compute the four performance measures as defined in Section 3.6. The accuracy analysis is reported in Table 2 and the precision in Table 3.

Table 2 reports the average over the seven experiments for each defect and in the bottom row, the ideal accuracy is reported based on the assumption that the CSLT would perfectly locate each defect.

The average effective area shows that for each defect the CSLT predicts that $76-83 \%$ of the area of the bore pile is consider to have good quality and be unaffected by defects. The size ratio for defects 1 and 2 is smaller than $100 \%$ which means that the actual defect is larger than the indicated size. On the contrary, for defect 3, the size is predicted to be nearly 2.5 times as large (243\%). This can be explained by Amir and Amir (2009); defects that are not in the middle of the pile, like defect 3, can appear larger on the CSLT results. On average defect 1 and defect 3 are located rather accurately, defect 1 is located within $28 \mathrm{~mm}$ of its actual location and defect 3 within $105 \mathrm{~mm}$ of its actual location. These distances are practically acceptable. For defect 2, the bandage on tube 3, the CSLT does not locate this defect accurately, it detects a potential defect at the centre of the pile. Recall from section 3.6 that the overlapping rate shows the portion of detected area that can be explained by the actual defect. Again defect 2 is not detected at the correct location, the detected areas for defect 1 and defect 3 are at least partially ( $85 \%$ and $35 \%)$ on the right location.

Table 2. Accuracy of CSLT (mean of seven repeated experiments).

\begin{tabular}{|c|c|c|c|c|}
\hline $\begin{array}{c}\text { Actual defect } \\
\text { depth(m) }\end{array}$ & $\begin{array}{c}\text { Average of } \\
\text { effective area } \\
(\%)\end{array}$ & $\begin{array}{c}\text { Average of } \\
\text { size ratio (\%) }\end{array}$ & $\begin{array}{c}\text { Average } \\
\text { of distance } \\
(\mathrm{mm})\end{array}$ & $\begin{array}{c}\text { Average of } \\
\text { overlapping } \\
\text { rate (\%) }\end{array}$ \\
\hline 1.0 & $76.7 \%$ & $81 \%$ & 28.1 & $85 \%$ \\
\hline 2.2 & $82.7 \%$ & $88.8 \% *$ & 512.3 & $0 \%$ \\
\hline 3.3 & $79.7 \%$ & $243 \%$ & 104.9 & $35 \%$ \\
\hline $\begin{array}{c}\text { Ideal } \\
\text { accuracy }\end{array}$ & $100 \%$ & $100 \%$ & 0 & $100 \%$ \\
\hline \multicolumn{6}{|c|}{ *Experiments without a detected defect were exlcuded. } \\
\hline
\end{tabular}

Table 3 reports the precision of the CSLT for each of the detected defect by reporting the standard deviation of the seven repeated measurements. The effective area is a rather imprecise performance measure. The other three performance measures which have small standard deviation
(StdDev) give more precise quantification of the defect. In general, the CSLT shows accurate and precise results over the seven experiments for defects 1 and 3 .

Table 3. Precision of CSLT (standard deviation of seven repeated experiments).

\begin{tabular}{|c|c|c|c|c|}
\hline $\begin{array}{c}\text { Defect } \\
\text { depth }(\mathrm{m})\end{array}$ & $\begin{array}{c}\text { StdDev of } \\
\text { effective area }\end{array}$ & $\begin{array}{c}\text { StdDev of } \\
\text { size ratio }\end{array}$ & $\begin{array}{c}\text { StdDev of } \\
\text { distance }\end{array}$ & $\begin{array}{c}\text { StdDev of } \\
\text { overlapping } \\
\text { rate }\end{array}$ \\
\hline 1.0 & 49 & 39 & 25.6 & 13 \\
\hline 2.2 & 160 & $66^{*}$ & 36.7 & - \\
\hline 3.3 & 170 & 16 & 17.7 & 3 \\
\hline \multicolumn{4}{|c}{$*$ Experiments without a detected defect were exlcuded. } \\
\hline
\end{tabular}

\subsubsection{Eight measurements during curing}

The accuracy and precision of the CSLT method after concreting. This is of interest as construction companies wish to perform the CSL and possibly CSLT test as quickly as possible after finishing concreting to save time and costs. In order to study this, eight CSLT measurements between one and 28 days after concreting (see section 3.4 for details) were taken. Figures 6-8 show the performance measures computed for each CSLT cross-sections for each defect plotted against time, where time is measured as days after concreting.

First consider the overlapping rate in Figure 6. It is observed that for defect 1 , the overlapping rate fluctuates and steadily increases with time as the pile cures the detected area overlaps more with the actual defect. Defect 2 is not detected at the right location and the detected area of defect 3 is a lot larger than the actual defect hence the overlapping rate stays rather low. Next consider Figure 7 the size ratio. Defect 2 in this figure is not reported as defect 2 was not identified at the right location. For defects 1 and 3 , it is observed that the size ratio fluctuates just after curing before coming down and stabilising around 1 for defect 1 , for defect 3 the size is over-estimated and it did not seem to be stabilised yet, but from Table 2 it is observed that it will go towards $2.43(243 \%)$. Figure 8 shows the location deviation between the detected defects and the actual defects. This stabilised around 12 days.

Overall Figures 6-8 show a similar pattern: in the beginning the measures are unstable and fluctuate to later stabilise. For overlapping rate and size ratio, stabilisation only seems to occur around the end of the measurement period, at the 28 day. However, the location of the defect is accurate measured around day 12. Concluding, for the bored pile the CSLT gives, two weeks after concreting, an accurate indication of the location of the defect, but the size and shape are still uncertain. However these results depend on the environment that the bored pile cured in. Based on the experimental results, it is recommended carrying out the CSL or CSLT test at a minimum of 14 days after concreting, and repeating the results over time to ensure accuracy of the detected defects. 


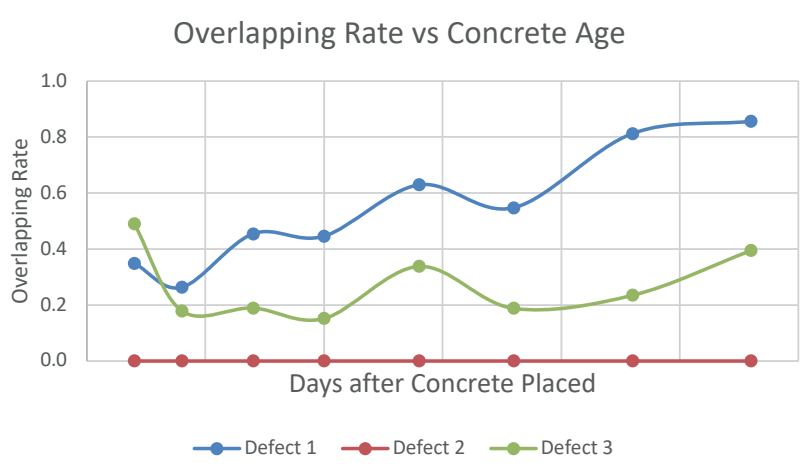

Figure 6 . The overlapping rate changes with time.

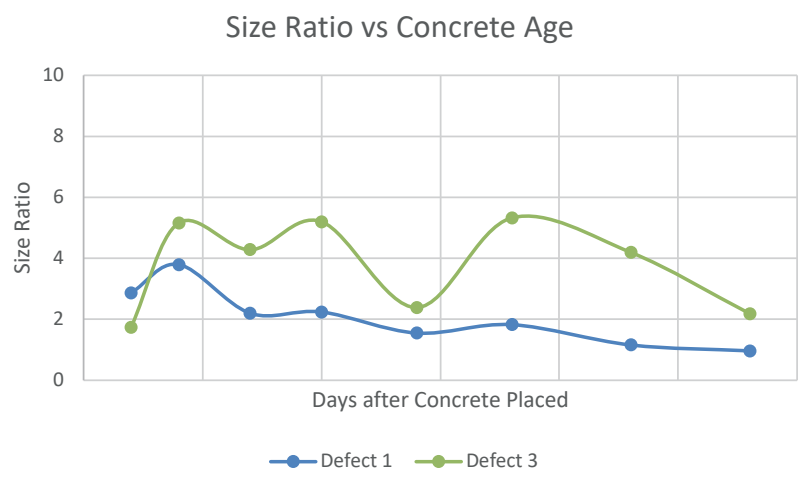

Figure 7. The size ratio changes with time.

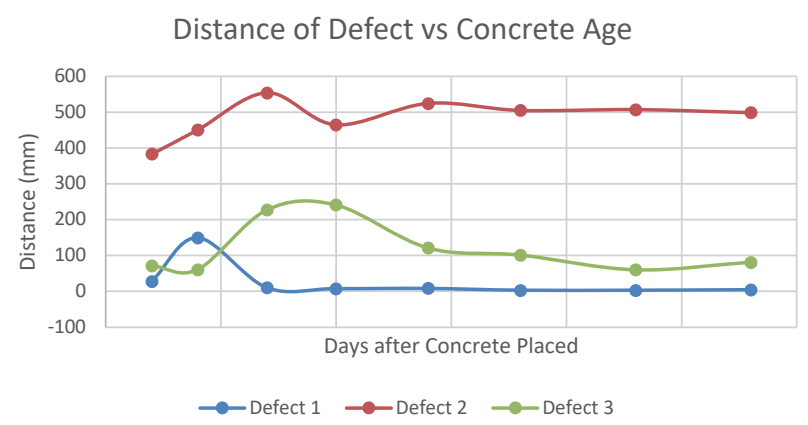

Figure 8 . The location deviation changes with time.

\section{Conclusion and discussion}

The accuracy and precision of the CSLT measurement system for bored pile testing were studied. The CSLT is currently not adopted in Hong Kong as an accredited test. A test pile with known defects were installed and 15 experiments were performed to obtain CSLT data.

Overall, the CSLT system provides accurate enough information regarding the location and size of the defects for practical use. It is recommended using the CSLT to diagnose defect identified by the CSL, especially when the measurements are performed at least 28 days after concreting. Based on the results of the study, the CSLT could be performed from 14 days after concreting, however only the location of the defect is accurately displayed by the CSLT at that curing stage. These results depend on the curing speed of the concrete and the type of defect materials in the pile.

Three defects in the test pile were placed. The first defect at $1 \mathrm{~m}$, a box in the centre of the pile, was accurately and precisely identified by the CSLT software. The second defect at $2.2 \mathrm{~m}$, a bandage on tube 3, was not detected correctly with the CSLT. However, this is practically not very problematic as it is only a small defect, and it therefore does not affect the structural integrity of the pile. The third defect at $3.3 \mathrm{~m}$ is also a box placed to the side of the pile. The CSLT estimated this as larger and more to the centre than it actually is. One possible reason could be the CSLT algorithm to construct 3D images. However, it is not due to the pile construction as the results are obtained using the as-built measurements. CSL does not tell us anything about defect size, hence CSLT will always be a useful additional source of information. However, it would be misleading that the size ratio can be larger than 1 , especially for defects out of the centre of the bored pile. This is something that the engineer needs to keep in mind.

The seven repeated measurements were in fact resulting in very visually similar and close tomography images, slight deviation of the images maybe caused by variation during the data collection. It is worthy to note that the CSLT procedure requires a considerable amount of time to perform especially for bored piles with six access tubes installed (15 profiles of CSLT scanning at each depth with anomaly detected). There is a huge amount of CSL data to be computed by the CSLT software. Slight difference between measurements such as the depth range and the depth steps may result in a slight variation of the result images.

A limitation to the CSLT method is that it cannot properly identify defects at the bottom of the pile. As the procedure of CSLT requires scanning of the defects in many different angles, including both below and above the actual defect depths making it impossible for the CSLT methods to be conducted for defect close to the bottom of the bored pile, which is also concluded by Hertlein and Davis (2006).

There are of course some limitations to this study. One limitation is that the test was conducted on a $1.2 \mathrm{~m}$ diameter test pile and that in Hong Kong generally bored piles are generally $3 \mathrm{~m}$ wide. Also, the pile is built above ground, this could have influence on the results obtained right after concreting, as curing of concrete will have different speeds above and below ground.

Future work could consider directly using the frequency to estimate defect size and location rather than relying on software of the equipment supplier. This may involve the study of the effects of the frequency of transducer used in the study (e.g. CHUM). Further study of the required depth range, depth step and suitable test timing when performing the CSLT will continue. In addition it would be interesting to study correlation between wave frequencies used in the test and the outcome. 


\section{Notes on contributors}

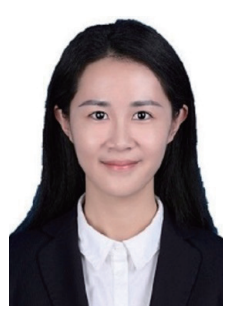

Ms Zezhong Wang is a Ph.D. student in the Department of Advanced Design and Systems Engineering at the City University of Hong Kong.

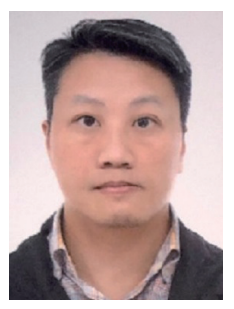

Mr Eric Tak Cho Ho is a Technical Manager in the Foundation Testing Department of the FT Laboratories Ltd. His research focuses on foundation testing and instrumentation monitoring.

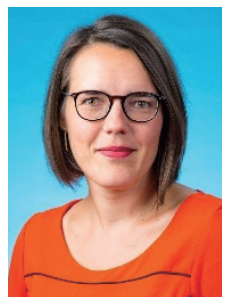

Dr Inez Maria Zwetsloot is an Assistant Professor in the Department of Advanced Design and Systems Engineering at the City University of Hong Kong. Her research focuses on statistical process monitoring and statistical engineering.

\section{References}

[1] Amir JM and Amir EI (2009). Capabilities and limitations of cross hole ultrasonic testing of piles. In Contemporary Topics in Deep Foundations, pp. 536543.

[2] American Society for Testing and Materials (ASTM) (2016). Standard test method for integrity testing of concrete deep foundations by ultrasonic crosshole testing. Designation D6760-16.

[3] Chernauskas LR and Hajduk EL (2009). The use of crosshole tomography to evaluate drilled shaft repairs. In Contemporary Topics in Deep Foundations, pp. 560-567.

[4] Sellountou AE, Amir JM, Canivan G, Chernauskas L, Hertlein B, Kandaris P, Kovacs T and Likins G (2019). Terminology and evaluation criteria of crosshole sonic logging (CSL) as applied to deep foundations. Deep Foundations Institute-PIMS Committee.

[5] Finno RJ and Champy P (1997). Cross-hole sonic logging evaluation of drilled shafts at the Northwestern University National Geotechnical Experimentation Site. Final Rep., Dept. of Civil Engineering, Northwestern Univ.

[6] Hajali M and Abishdid C (2014). Cross-hole sonic logging and frequency tomography analysis of drilled shaft foundations to better evaluate anomalies locations. DFI Journal-The Journal of the Deep Foundations Institute, 8(1), pp.27-38.
[7] Hajduk EL, Tallent MR, Ledford DL and Christopher WR (2004). Crosshole sonic logging integrity testing for the new cooper river bridge. International Conference on Case Histories in Geotechnical Engineering.

[8] Islam MS, Yasmin T, Hannan SA and Peela P (2015). Integrity evaluation of concrete by cross-hole sonic logging in Bangladesh. IABSE-JSCE Joint Conference on Advances in Bridge Engineering-III.

[9] Jalinoos F, Mekic N and Hanna K (2005). Drilled shaft foundation defects: identification, imaging, and characterization. FHWA, Publication No. FHWACFL/TD-05-007.

[10] Leung C (2017). Officers arrest 21 over faked concrete test results for Hong Kong-Zhuhai-Macau bridge project. South China Morning Post. [online]. Available at: <https://www.scmp.com/news/hongkong/law-crime/article/2095389/officers-arrest-21over-faked-concrete-test-results-hong $>$.

[11] Likins G, Robinson B and Piscsalko G (2012). A brief overview of testing of deep foundations. Proceedings, Testing and Design Methods for Deep Foundations, Kanazawa, Japan, pp.97-104.

[12] Likins G, Rausche F, Webster K and Klesney A (2007). Defect analysis for CSL testing. In Contemporary Issues in Deep Foundations, pp. 1-10.

[13] Lozovsky IN, Churkin AA and Zhostkov RA (2020). Localization of defects in bored pile physical model using cross-hole ultrasonic tomography. Bulletin of the Russian Academy of Sciences: Physics, 84(2), pp.215219.

[14] Mor G (1991). Fault detecting in foundation piles and sheet walls with ultrasonic investigations. In 4th International Conference on Piling and Deep Foundations, Balkema of Rotterdam, Vol. 1, pp. 7-12.

[15] Sibit D and Handayani G (2016). The Crosshole Sonic Logging (CSL) Measurement System to Measure the Quality of Physical Model of Bored Pile. In Journal of Physics: Conference Series, Vol. 739, No. 1, p. 012051.

[16] Williams HT and Jones I (2008). Interpretation and misinterpretation of crosshole sonic logging test results. In Deep Foundations on Bored and Auger Piles-BAP V, pp. 311-316.

[17] White B, Nagy M and Allin R (2008). Comparing cross-hole sonic logging and low-strain integrity testing results. In Proceedings of the Eighth International Conference on the Application of Stress Wave Theory to Piles, pp. 471-476.

[18] Zhussupbekov A, Morev I, Tanyrbergenova G and Shakirova N (2019). Evaluation of the quality of pile foundations by different methods. In MATEC Web of Conferences, Vol. 265, p. 05013. 